\title{
Periodic boundary value problems for impulsive neutral differential equations with multi-deviation arguments
}

\author{
GUOBING YE ${ }^{1 *}$, JIANHUA SHEN ${ }^{2}$ and JIANLI $\mathrm{LI}^{3}$ \\ ${ }^{1}$ College of Science, Hunan University of Technology, Zhuzhou Hunan 412008, PR, China \\ ${ }^{2}$ College of Science, Hangzhou Normal University, Hangzhou Zhejiang 310036, PR, China \\ ${ }^{3}$ College of Mathematics and Computer Science, Hunan Normal University \\ Changsha Hunan 410081, PR, China \\ E-mails: yeguobing19@sina.com / jhshen2ca@yahoo.com / 1jianli@sina.com
}

\begin{abstract}
We develop the impulsive inequality and the classical lower and upper solutions, and establish the comparison principles. By using these results and the monotone iterative technique, we obtain the existence of solutions of periodic boundary value problems for a class of impulsive neutral differential equations with multi-deviation arguments. An example is given to demonstrate our main results.
\end{abstract}

Mathematical subject classification: Primary: 34A37; Secondary: 34k10.

Key words: Impulsive neutral differential equation, periodic boundary value, impulsive inequality, lower and upper solutions, monotone iterative technique, extremal solution.

\section{Introduction}

Impulsive differential equations have become more important in recent years in some mathematical models of real phenomena, especially in control, biological or medical domains (see, for example, [1-5]). As to periodic boundary value problems for impulsive differential equations, many authors have obtained

\#CAM-136/09. Received: 02/X/09. Accepted: 18/V/10.

*The first author is supported by the NNSF of China (n. 10871062). 
excellent existence results; see, for instance, [7-11] for impulsive differential equations, [15-18] for impulsive neutral functional differential equations, [19] for abstract impulsive neutral functional differential equations. In [15-19], the characters of their neutral types are

$$
\begin{aligned}
& \frac{d}{d t}\left[x(t)-g\left(t, x_{t}\right)\right], \quad \frac{d}{d t}\left(u(t)+g\left(t, u_{t}\right)\right), \quad \frac{d}{d t}\left(u(t)+F\left(t, u_{t}\right)\right), \\
& \frac{d}{d t}\left[x(t)-g\left(t, x_{t}, \int_{0}^{t} a\left(t, s, x_{s}\right) d s\right)\right] \quad \text { and } \quad \frac{d^{2}}{d t^{2}}\left(x(t)-g\left(t, x_{t}\right)\right),
\end{aligned}
$$

respectively. In this paper, however, the character of its neutral type is $(u(\theta(t)))^{\prime}$. The character is different from the previous ones. Consider the following periodic boundary value problems for impulsive neutral differential equations with multi-deviation arguments of the form

$$
\left\{\begin{array}{l}
(u(\theta(t)))^{\prime}=f\left(t, u(t), u\left(\varphi_{1}(t)\right), \ldots, u\left(\varphi_{q}(t)\right)\right), \\
t \in J=[0, T], t \neq \zeta_{k}, \\
\Delta u\left(t_{k}\right)=I_{k}\left(u\left(t_{k}\right)\right), \quad k=1, \ldots, p, \\
u(0)=u(T),
\end{array}\right.
$$

where $0=t_{0}<t_{1}<\cdots<t_{p}<t_{p+1}=T$; $\theta \in C^{1}(J, \mathbb{R}), \theta$ is monotone increasing with $0 \leq \theta(t) \leq t(t \in J), \theta(0)=0, \theta(T)=T$, and set $\theta\left(\zeta_{k}\right)=t_{k}(k=1, \ldots, p), J_{0}=J \backslash\left\{t_{1}, \ldots, t_{p}\right\}, J_{1}=J \backslash\left\{\zeta_{1}, \ldots, \zeta_{p}\right\} ; f: J \times$ $\mathbb{R}^{q+1} \rightarrow \mathbb{R}$ is continuous almost everywhere, and $\varphi_{i}: J \rightarrow \mathbb{R}$ continuous with $\varphi_{i}(J) \subseteq J(i=1, \ldots, q)$; and $I_{k} \in C(\mathbb{R}, \mathbb{R}), \Delta u\left(t_{k}\right)=u\left(t_{k}^{+}\right)-$ $u\left(t_{k}\right)$. Denote by $P C(X, Y)$, where $X \subset \mathbb{R}, Y \subset \mathbb{R}$, the set of all functions $u: X \rightarrow Y$ which are piecewise continuous in $X$ with points of discontinuity of the first kind at the points $t_{k} \in X$, i.e., there exist the limits $u\left(t_{k}^{+}\right)<\infty$ and $u\left(t_{k}^{-}\right)=u\left(t_{k}\right)<\infty . P C^{1}(X, Y)$ denotes the set of all functions $u \in P C(X, Y)$, that are continuously differentiate for $t \in X, t \neq t_{k}$. Let $\Omega=P C([0, T], \mathbb{R}) \bigcap P C^{1}([0, T], \mathbb{R})$.

Definition. We say that the functions $\alpha, \beta \in \Omega$ are lower and upper solutions of (1.1), respectively, if there exist $M>0$ and $0 \leq L_{k}<1$ such that

$$
\left\{\begin{array}{l}
(\alpha(\theta(t)))^{\prime} \leq f\left(t, \alpha(t), \alpha\left(\varphi_{1}(t)\right), \ldots, \alpha\left(\varphi_{q}(t)\right)\right)-a(t), \quad t \in J_{1}, \\
\Delta \alpha\left(t_{k}\right) \leq I_{k}\left(\alpha\left(t_{k}\right)\right)-L_{k} a_{k}, \quad k=1, \ldots, p,
\end{array}\right.
$$


where

$$
\begin{aligned}
& a(t)= \begin{cases}0, & \alpha(0) \leq \alpha(T), \\
\frac{\theta^{\prime}(t)+M t+\sum_{i=1}^{q} N_{i} \varphi_{i}(t)}{T}(\alpha(0)-\alpha(T)), & \alpha(0)>\alpha(T),\end{cases} \\
& a_{k}= \begin{cases}0, & \alpha(0) \leq \alpha(T), \\
\frac{t_{k}}{T}(\alpha(0)-\alpha(T)), & \alpha(0)>\alpha(T),\end{cases}
\end{aligned}
$$

and

$$
\left\{\begin{array}{l}
(\beta(\theta(t)))^{\prime} \geq f\left(t, \beta(t), \beta\left(\varphi_{1}(t)\right), \ldots, \beta\left(\varphi_{q}(t)\right)\right)+b(t), \quad t \in J_{1}, \\
\Delta \beta\left(t_{k}\right) \geq I_{k}\left(\beta\left(t_{k}\right)\right)+L_{k} b_{k}, \quad k=1, \ldots, p,
\end{array}\right.
$$

where

$$
\begin{aligned}
& b(t)= \begin{cases}0, & \beta(0) \geq \beta(T), \\
\frac{\theta^{\prime}(t)+M t+\sum_{i=1}^{q} N_{i} \varphi_{i}(t)}{T}(\beta(T)-\beta(0)), & \beta(0)<\beta(T),\end{cases} \\
& b_{k}= \begin{cases}0, & \beta(0) \geq \beta(T), \\
\frac{t_{k}}{T}(\beta(T)-\beta(0)), & \beta(0)<\beta(T) .\end{cases}
\end{aligned}
$$

The definitions of classical lower and upper solutions make reference to the case $\alpha(0) \leq \alpha(T)$ and $\beta(0) \geq \beta(T)$.

\section{Preliminaries}

Lemma 1. Let $s \in[0, T], c_{k} \geq 0, \alpha_{k}, k=1, \ldots, p$ be constants, $p, q \in$ $P C(J, \mathbb{R}), x \in P C^{1}(J, \mathbb{R})$ and $\theta$ be set by $(1.1)$. If

$$
\begin{cases}(x(\theta(t)))^{\prime} \leq p(t) x(\theta(t))+q(t), & t \in[s, T), t \neq \zeta_{k}, \\ x\left(t_{k}^{+}\right) \leq c_{k} x\left(t_{k}\right)+\alpha_{k}, & t_{k} \in[s, T),\end{cases}
$$

then for $t \in[s, T]$,

$$
\begin{aligned}
& x(\theta(t)) \leq x\left(\theta\left(s^{+}\right)\right)\left(\prod_{s<\zeta_{k}<t} c_{k}\right) \exp \left(\int_{s}^{t} p(u) d u\right)+\int_{s}^{t}\left(\prod_{u<\zeta_{k}<t} c_{k}\right) \\
& \times \exp \left(\int_{u}^{t} p(\tau) d \tau\right) q(u) d u+\sum_{s<\zeta_{k}<t}\left(\prod_{\zeta_{k}<\zeta_{i}<t} c_{i}\right) \exp \left(\int_{\zeta_{k}}^{t} p(\tau) d \tau\right) \alpha_{k} .
\end{aligned}
$$


This proof is similar to the one of [1], here we omit it.

Lemma 2. Let $u \in \Omega, M>0, N_{i} \geq 0(i=1, \ldots, q), 0 \leq L_{k}<1$ and $\theta$ be set by (1.1), such that

(A1) $(u(\theta(t)))^{\prime}+M u(t)+\sum_{i=1}^{q} N_{i} u\left(\varphi_{i}(t)\right) \leq 0, \quad t \in J_{1}$;

(A2) $\Delta u\left(t_{k}\right) \leq-L_{k} u\left(t_{k}\right), \quad k=1, \ldots, p$;

(A3) $u(0) \leq u(T)$;

(A4) $\left(M+\sum_{i=1}^{q} N_{i}\right) \int_{0}^{T} \prod_{s<\zeta_{k}<T}\left(1-L_{k}\right) d s \leq \prod_{k=1}^{p}\left(1-L_{k}\right)^{2}$.

Then $u \leq 0$ on $J$.

Proof. By (A1) and (A2), we have

$$
\begin{aligned}
& (u(\theta(t)))^{\prime} \leq-M u(t)-\sum_{i=1}^{q} N_{i} u\left(\varphi_{i}(t)\right), \quad t \in J_{1}, \\
& \frac{d u(\tau)}{d \tau} \cdot \frac{d \tau}{d t} \leq-M u(t)-\sum_{i=1}^{q} N_{i} u\left(\varphi_{i}(t)\right), \quad t \in J_{1}, \tau=\theta(t), \\
& u\left(t_{k}^{+}\right) \leq\left(1-L_{k}\right) u\left(t_{k}\right), \quad k=1, \ldots, p .
\end{aligned}
$$

To prove $u(t) \leq 0$ on $J$, we shall consider the following two cases.

Case 1. $u(t) \geq 0$ for all $t \in J$. In this case, by (2.2) and (2.3) and the properties of $\theta$, we get $u^{\prime}(t) \leq 0$ on $J_{0}$ and $u\left(t_{k}^{+}\right) \leq u\left(t_{k}\right), k=1, \ldots, p$. Therefore $u(t)$ is a non-increasing function on $J$. Then $u(0) \geq u(T)$. Since $u(0) \leq u(T), u(t) \equiv c$ on $J$ ( $c$ is a non-negative constant), and $u^{\prime}=0$ on $J_{0}$. This and (A1) imply $\left(M+\sum_{i=1}^{q} N_{i}\right) c \leq 0$. Then $u \equiv 0$ on $J$.

Case 2. There exists $t^{*} \in J$ such that $u\left(t^{*}\right)>0$ and $u(t)$ can take negative values in $J$. Let $\theta\left(\zeta^{*}\right)=t^{*}$. Again let $\bar{\zeta}=\min \left\{t \in J, \inf _{s \in J} u(\theta(s))=\right.$ $u(\theta(t))=-\lambda, \lambda>0\}$. Since (A3), $\bar{\zeta} \in[0, T)$. Without loss of generality, let $\bar{\zeta} \neq \zeta_{k}, \zeta_{k}^{+}, k=1, \ldots, p$ (If $\bar{\zeta}=\zeta_{k}$ or $\zeta_{k}^{+}$, the proof is similar, here we omit.). In this case, we consider two subcases. 
Subcase 1. $u(T)>0$. From (2.1), we have

$$
(u(\theta(t)))^{\prime} \leq \lambda\left(M+\sum_{i=1}^{q} N_{i}\right), \quad t \in J_{1} .
$$

In view of (2.4), (2.3), and Lemma 1, we can get for $t \in[\bar{\zeta}, T]$,

$$
\begin{aligned}
u(\theta(t)) & \leq u(\theta(\bar{\zeta})) \prod_{\bar{\zeta}<\zeta_{k}<t}\left(1-L_{k}\right)+\int_{\bar{\zeta}}^{t} \prod_{s<\zeta_{k}<t}\left(1-L_{k}\right) \lambda\left(M+\sum_{i=1}^{q} N_{i}\right) d s \\
& =-\lambda \prod_{\bar{\zeta}<\zeta_{k}<t}\left(1-L_{k}\right)+\lambda\left(M+\sum_{i=1}^{q} N_{i}\right) \int_{\bar{\zeta}}^{t} \prod_{s<\zeta_{k}<t}\left(1-L_{k}\right) d s .
\end{aligned}
$$

Let $t=T$. Then we have

$$
\begin{aligned}
u(\theta(T)) & \leq-\lambda \prod_{\bar{\zeta}<\zeta_{k}<T}\left(1-L_{k}\right)+\lambda\left(M+\sum_{i=1}^{q} N_{i}\right) \int_{\bar{\zeta}}^{T} \prod_{s<\zeta_{k}<T}\left(1-L_{k}\right) d s, \\
u(T) & \leq \lambda\left[\left(M+\sum_{i=1}^{q} N_{i}\right) \int_{0}^{T} \prod_{s<\zeta_{k}<T}\left(1-L_{k}\right) d s-\prod_{k=1}^{p}\left(1-L_{k}\right)\right] .
\end{aligned}
$$

Since $u(T)>0$, we get

$$
\begin{aligned}
& \prod_{k=1}^{p}\left(1-L_{k}\right)<\left(M+\sum_{i=1}^{q} N_{i}\right) \int_{0}^{T} \prod_{s<\zeta_{k}<T}\left(1-L_{k}\right) d s, \\
& \prod_{k=1}^{p}\left(1-L_{k}\right)^{2}<\left(M+\sum_{i=1}^{q} N_{i}\right) \int_{0}^{T} \prod_{s<\zeta_{k}<T}\left(1-L_{k}\right) d s,
\end{aligned}
$$

which is contradictory to (A4).

Subcase 2. $u(T) \leq 0$. In this subcase, then $\bar{\zeta}<\zeta^{*}$ or $\bar{\zeta}>\zeta^{*}$.

(i) $\bar{\zeta}<\zeta^{*}$. According to the same arguments as (2.5), we get

$$
\begin{aligned}
u\left(\theta\left(\zeta^{*}\right)\right) \leq & -\lambda \prod_{\bar{\zeta}<\zeta_{k}<\zeta^{*}}\left(1-L_{k}\right)+\lambda\left(M+\sum_{i=1}^{q} N_{i}\right) \\
& \times \int_{\bar{\zeta}}^{\zeta^{*}} \prod_{s<\zeta_{k}<\zeta^{*}}\left(1-L_{k}\right) d s .
\end{aligned}
$$


Multiplying both sides of the above inequality by $\prod_{\zeta^{*} \leq \zeta_{k}<T}\left(1-L_{k}\right)$ (If $\zeta^{*}>\zeta_{p}$, this reduction is not needed.), we obtain

$$
\begin{aligned}
& u\left(\theta\left(\zeta^{*}\right)\right) \prod_{\zeta^{*} \leq \zeta_{k}<T}\left(1-L_{k}\right) \\
& \quad \leq-\lambda \prod_{\bar{\zeta}<\zeta_{k}<T}\left(1-L_{k}\right)+\lambda\left(M+\sum_{i=1}^{q} N_{i}\right) \int_{\bar{\zeta}}^{\zeta^{*}} \prod_{s<\zeta_{k}<T}\left(1-L_{k}\right) d s \\
& \quad \leq-\lambda \prod_{k=1}^{p}\left(1-L_{k}\right)+\lambda\left(M+\sum_{i=1}^{q} N_{i}\right) \int_{0}^{T} \prod_{s<\zeta_{k}<T}\left(1-L_{k}\right) d s \\
& \quad=\lambda\left[\left(M+\sum_{i=1}^{q} N_{i}\right) \int_{0}^{T} \prod_{s<\zeta_{k}<T}\left(1-L_{k}\right) d s-\prod_{k=1}^{p}\left(1-L_{k}\right)\right] .
\end{aligned}
$$

Since $u\left(\theta\left(\zeta^{*}\right)\right)>0$, we have

$$
\begin{aligned}
& \prod_{k=1}^{p}\left(1-L_{k}\right)<\left(M+\sum_{i=1}^{q} N_{i}\right) \int_{0}^{T} \prod_{s<\zeta_{k}<T}\left(1-L_{k}\right) d s, \\
& \prod_{k=1}^{p}\left(1-L_{k}\right)^{2}<\left(M+\sum_{i=1}^{q} N_{i}\right) \int_{0}^{T} \prod_{s<\zeta_{k}<T}\left(1-L_{k}\right) d s,
\end{aligned}
$$

which is contradictory to (A4).

(ii) $\bar{\zeta}>\zeta^{*}$. By (A3), we have $u(0) \leq 0$. This and the properties of $\theta$ imply $0<\zeta^{*}$. According to the same arguments as (2.5), we get

$$
\begin{aligned}
u\left(\theta\left(\zeta^{*}\right)\right) \leq & u(\theta(0)) \prod_{0<\zeta k<\zeta^{*}}\left(1-L_{k}\right) \\
& +\lambda\left(M+\sum_{i=1}^{q} N_{i}\right) \int_{0}^{\zeta^{*}} \prod_{s<\zeta \zeta_{k}<\zeta^{*}}\left(1-L_{k}\right), \\
u\left(\theta\left(\zeta^{*}\right)\right) \leq & u(T) \prod_{0<\zeta \zeta_{k}<\zeta^{*}}\left(1-L_{k}\right) \\
& +\lambda\left(M+\sum_{i=1}^{q} N_{i}\right) \int_{0}^{\zeta^{*}} \prod_{s<\zeta \zeta_{k}<\zeta^{*}}\left(1-L_{k}\right) d s .
\end{aligned}
$$

Since $u\left(\theta\left(\zeta^{*}\right)\right)>0$, we have

$$
u(T) \prod_{0<\zeta \zeta_{k}<\zeta^{*}}\left(1-L_{k}\right) \geq-\lambda\left(M+\sum_{i=1}^{q} N_{i}\right) \int_{0}^{\zeta^{*}} \prod_{s<\zeta_{k}<\zeta^{*}}\left(1-L_{k}\right) d s .
$$


By (2.5) and (2.6), we obtain

$$
\begin{gathered}
\prod_{0<\zeta_{k}<\zeta^{*}}\left(1-L_{k}\right) \prod_{\bar{\zeta}<\zeta k<T}\left(1-L_{k}\right) \leq\left(M+\sum_{i=1}^{q} N_{i}\right) \\
\times\left[\int_{0}^{\zeta^{*}} \prod_{s<\zeta_{k}<\zeta^{*}}\left(1-L_{k}\right) d s+\prod_{0<\zeta_{k}<\zeta^{*}}\left(1-L_{k}\right) \int_{\bar{\zeta}}^{T} \prod_{s<\zeta_{k}<T}\left(1-L_{k}\right) d s\right] .
\end{gathered}
$$

Multiplying both sides of the above inequality by $\prod_{\zeta^{*} \leq \zeta_{k}<T}\left(1-L_{k}\right)$ (If $\zeta^{*}>\zeta_{p}$, this reduction is not needed.), we get

$$
\begin{aligned}
& \prod_{0<\zeta_{k}<T}\left(1-L_{k}\right) \prod_{\bar{\zeta}<\zeta_{k}<T}\left(1-L_{k}\right) \\
& \leq\left(M+\sum_{i=1}^{q} N_{i}\right)\left[\prod_{\zeta^{*} \leq \zeta_{k}<T}\left(1-L_{k}\right) \int_{0}^{\zeta^{*}} \prod_{s<\zeta_{k}<\zeta^{*}}\left(1-L_{k}\right) d s\right. \\
& \left.\quad+\prod_{0<\zeta_{k}<T}\left(1-L_{k}\right) \int_{\bar{\zeta}}^{T} \prod_{s<\zeta_{k}<T}\left(1-L_{k}\right) d s\right], \\
& \prod_{k=1}^{p}\left(1-L_{k}\right) \prod_{\bar{\zeta}<\zeta_{k}<T}\left(1-L_{k}\right) \\
& \quad \leq\left(M+\sum_{i=1}^{q} N_{i}\right)\left[\int_{0}^{\zeta^{*}} \prod_{s<\zeta_{k}<T}\left(1-L_{k}\right) d s+\int_{\bar{\zeta}}^{T} \prod_{s<\zeta_{k}<T}\left(1-L_{k}\right) d s\right], \\
& \prod_{k=1}^{p}\left(1-L_{k}\right) \prod_{\bar{\zeta}<\zeta_{k}<T}\left(1-L_{k}\right)<\left(M+\sum_{i=1}^{q} N_{i}\right) \int_{0}^{T} \prod_{s<\zeta_{k}<T}\left(1-L_{k}\right) d s, \\
& \prod_{k=1}^{p}\left(1-L_{k}\right)^{2}<\left(M+\sum_{i=1}^{q} N_{i}\right) \int_{0}^{T} \prod_{s<\zeta_{k}<T}\left(1-L_{k}\right) d s,
\end{aligned}
$$

which is contradictory to (A4).

Thus, in either case $u \leq 0$ on $J$. Therefore the proof of the Lemma is complete.

Lemma 3. Let $u \in \Omega, M>0, N_{i} \geq 0(i=1, \ldots, q), 0 \leq L_{k}<1$ and $\theta$ be set by (1.1), such that 
(B1) $(u(\theta(t)))^{\prime}+M u(t)+\sum_{i=1}^{q} N_{i} u\left(\varphi_{i}(t)\right)+\frac{\theta^{\prime}(t)+M t+\sum_{i=1}^{q} N_{i} \varphi_{i}(t)}{T}[u(0)-u(T)] \leq$ $0, t \in J_{1}$;

(B2) $\Delta u\left(t_{k}\right) \leq-L_{k} u\left(t_{k}\right)-L_{k} \times \frac{t_{k}}{T}[u(0)-u(T)], \quad k=1, \ldots, p$;

(B3) $u(0)>u(T)$.

Also assume that (A4) holds. Then $u \leq 0$ on $J$.

Proof. Set $m(t)=u(t)+t / T \cdot[u(0)-u(T)]$. Clearly, $m(0)=m(T)$. It follows that for $t \in J_{0}$,

$$
\begin{gathered}
(m(\theta(t)))^{\prime}+M m(t)+\sum_{i=1}^{q} N_{i} m\left(\varphi_{i}(t)\right)=(u(\theta(t)))^{\prime}+M u(t) \\
+\sum_{i=1}^{q} N_{i} u\left(\varphi_{i}(t)\right)+\frac{\theta^{\prime}(t)+M t+\sum_{i=1}^{q} N_{i} \varphi_{i}(t)}{T}[u(0)-u(T)] \leq 0
\end{gathered}
$$

and for $t=t_{k}$,

$$
\Delta m\left(t_{k}\right)=\Delta u\left(t_{k}\right) \leq-L_{k} u\left(t_{k}\right)-L_{k} \times t_{k} / T \cdot[u(0)-u(T)]=-L_{k} m\left(t_{k}\right) .
$$

By Lemma 2, we obtain $m(t) \leq 0$ on $J$, and so $u(t) \leq 0$ on $J$. Thus, we have completed the proof of the Lemma.

\section{Existence for linear problem}

In this section, we consider the linear problem of (1.1)

$$
\left\{\begin{array}{l}
(u(\theta(t)))^{\prime}+M u(t)+\sum_{i=1}^{q} N_{i} u\left(\varphi_{i}(t)\right)=\sigma(t), \quad t \in J_{1}, \\
\Delta u\left(t_{k}\right)=-L_{k} u\left(t_{k}\right)+\gamma_{k}, \quad k=1, \ldots, p \\
u(0)=u(T)
\end{array}\right.
$$

where $\sigma(t) \in P C(J, \mathbb{R}), \gamma_{k} \in \mathbb{R}, k=1, \ldots, p, M>0, N_{i} \geq 0(i=$ $1, \ldots, q), 0 \leq L_{k}<1$ and $\theta$ is set by (1.1). For $\alpha, \beta \in \Omega$, set $[\alpha, \beta]=$ $\{u \mid \alpha(t) \leq u(t) \leq \beta(t), t \in J\}$. 
Theorem 1. Suppose that there exist $\alpha, \beta \in \Omega$ such that

(C1) $\alpha \leq \beta$ on $J$

(C2) $(\alpha(\theta(t)))^{\prime}+M \alpha(t)+\sum_{i=1}^{q} N_{i} \alpha\left(\varphi_{i}(t)\right) \leq \sigma(t)-a(t), \quad t \in J_{1}$, $\Delta \alpha\left(t_{k}\right) \leq-L_{k} \alpha\left(t_{k}\right)+\gamma_{k}-a_{k}, \quad k=1, \ldots, p ;$ $(\beta(\theta(t)))^{\prime}+M \beta(t)+\sum_{i=1}^{q} N_{i} \beta\left(\varphi_{i}(t)\right) \geq \sigma(t)+b(t), \quad t \in J_{1}$, $\Delta \beta\left(t_{k}\right) \geq-L_{k} \beta\left(t_{k}\right)+\gamma_{k}+b_{k}, \quad k=1, \ldots, p$, where $a(t), b(t), a_{k}, b_{k}$ are defined by (1.2)-(1.5). Also assume that (A4) holds. Then there exists a unique solution $u$ for (3.1) with $u \in[\alpha, \beta]$.

Proof. We shall prove the Theorem in the following three steps.

Step 1. If $u_{1}, u_{2}$ are solutions of (3.1), set $v_{1}=u_{1}-u_{2}$ and $v_{2}=u_{2}-u_{1}$, then

$$
\left\{\begin{array}{l}
\left(v_{1}(\theta(t))\right)^{\prime}+M v_{1}(t)+\sum_{i=1}^{q} N_{i} v_{1}\left(\varphi_{i}(t)\right)=0, \quad t \in J_{1}, \\
\Delta v_{1}\left(t_{k}\right)=-L_{k} v_{1}\left(t_{k}\right), \quad k=1, \ldots, p, \\
v_{1}(0)=v_{1}(T),
\end{array}\right.
$$

and

$$
\left\{\begin{array}{l}
\left(v_{2}(\theta(t))\right)^{\prime}+M v_{2}(t)+\sum_{i=1}^{q} N_{i} v_{2}\left(\varphi_{i}(t)\right)=0, \quad t \in J_{1}, \\
\Delta v_{2}\left(t_{k}\right)=-L_{k} v_{2}\left(t_{k}\right), \quad k=1, \ldots, p, \\
v_{2}(0)=v_{2}(T) .
\end{array}\right.
$$

By Lemma 2, we obtain $v_{1}=u_{1}-u_{2} \leq 0$ and $v_{2}=u_{2}-u_{1} \leq 0$. Thus $u_{1}=u_{2}$. Then there exists a unique solution $u$ for (3.1).

Step 2. We prove that if $\omega, \gamma$ are classical lower and upper solutions, respectively, for (3.1) with $\omega \leq \gamma$, then (3.1) has a solution $u \in[\omega, \gamma]$.

Let $u(\cdot, a)$ denote the unique solution of the following equation

$$
\left\{\begin{array}{l}
(u(\theta(t)))^{\prime}+M u(t)+\sum_{i=1}^{q} N_{i} u\left(\varphi_{i}(t)\right)=\sigma(t), \quad t \in J_{1}, \\
\Delta u\left(t_{k}\right)=-L_{k} u\left(t_{k}\right)+\gamma_{k}, \quad k=1, \ldots, p, \\
u(0)=a .
\end{array}\right.
$$


Firstly, we show $\omega(0) \leq u(T, \omega(0))$ and $\gamma(0) \geq u(T, \gamma(0))$. Assume $\omega(0)>u(T, \omega(0))$. Let $v(t)=\omega(t)-u(t, \omega(0))$. Then the function $v$ satisfies

$$
\left\{\begin{array}{l}
(v(\theta(t)))^{\prime}+M v(t)+\sum_{i=1}^{q} N_{i} v\left(\varphi_{i}(t)\right) \leq 0, \quad t \in J_{1}, \\
\Delta v\left(t_{k}\right)=-L_{k} v\left(t_{k}\right), \quad k=1, \ldots, p, \\
v(0)=\omega(0)-\omega(0)<\omega(T)-u(T, \omega(0))=v(T) .
\end{array}\right.
$$

By Lemma 2, we have $v(t) \leq 0$ on $J$. This implies $v(T)=\omega(T)-u(T, \omega(0)) \leq$ 0 . Thus $\omega(0) \leq \omega(T) \leq u(T, \omega(0))$, which is contradictory to the above assumption. Then $\omega(0) \leq u(T, \omega(0))$. Similarly, we have $\gamma(0) \geq u(T, \gamma(0))$.

Next, we prove that there exists $c \in[\omega(0), \gamma(0)]$ such that $u(0, c)=u(T, c)$. Now, we consider two cases.

Case 1. $\omega(0)=\gamma(0)$. In this case, we get $\omega(0) \leq u(T, \gamma(0)) \leq \gamma(0)=\omega(0)$. Thus $u(T, \gamma(0))=\omega(0)$. Then we choose $c=\omega(0)$, and so $u=u(\cdot, c)$ is a solution of (3.1).

Case 2. $\omega(0)<\gamma(0)$. In this case, we define the map $F:[\omega(0), \gamma(0)] \rightarrow \mathbb{R}$ by $F(s)=s-u(T, s)$. Clearly $F$ is continuous. Since $F(\omega(0)) \leq 0 \leq F(\gamma(0))$, there must exist one point $c \in[\omega(0), \gamma(0)]$ such that $F(c)=0$. Then $u=u(\cdot, c)$ is a solution of (3.1).

Finally, we claim $u \in[\omega, \gamma]$. Let $m_{1}(t)=\omega(t)-u(t, c)$ and $m_{2}(t)=$ $u(t, c)-\gamma(t)$. It is evident that $m_{1}, m_{2} \in \Omega$, and

$$
\left\{\begin{array}{l}
\left(m_{1}(\theta(t))\right)^{\prime}+M m_{1}(t)+\sum_{i=1}^{q} N_{i} m_{1}\left(\varphi_{i}(t)\right) \leq 0, \quad t \in J_{1}, \\
\Delta m_{1}\left(t_{k}\right) \leq-L_{k} m_{1}\left(t_{k}\right), \quad k=1, \ldots, p, \\
m_{1}(0)=\omega(0)-u(0, c) \leq \omega(T)-u(T, c)=m_{1}(T),
\end{array}\right.
$$

and

$$
\left\{\begin{array}{l}
\left(m_{2}(\theta(t))\right)^{\prime}+M m_{2}(t)+\sum_{i=1}^{q} N_{i} m_{2}\left(\varphi_{i}(t)\right) \leq 0, \quad t \in J_{1}, \\
\Delta m_{2}\left(t_{k}\right) \leq-L_{k} m_{2}\left(t_{k}\right), \quad k=1, \ldots, p, \\
m_{2}(0)=u(0, c)-\gamma(0) \leq u(T, c)-\gamma(T)=m(T) .
\end{array}\right.
$$

Using Lemma 2, we obtain $m_{1} \leq 0$ and $m_{2} \leq 0$ on $J$. Thus $\omega \leq u(\cdot, c) \leq \gamma$ on $J$. 
Step 3. We prove that $\bar{\alpha}(t), \bar{\beta}(t)$ are classical lower and upper solutions, respectively, for (3.1) with $\bar{\alpha} \leq \bar{\beta}$, moreover $[\bar{\alpha}, \bar{\beta}] \subseteq[\alpha, \beta]$, where

$$
\bar{\alpha}(t)= \begin{cases}\alpha(t), & \alpha(0) \leq \alpha(T), \\ \alpha(t)+t / T \cdot[\alpha(0)-\alpha(T)], & \alpha(0)>\alpha(T),\end{cases}
$$

and

$$
\bar{\beta}(t)= \begin{cases}\beta(t), & \beta(0) \geq \beta(T), \\ \beta(t)-t / T \cdot[\beta(T)-\beta(0)], & \beta(0)<\beta(T) .\end{cases}
$$

It is evident that $\alpha \leq \bar{\alpha}$ and $\bar{\beta} \leq \beta$ on $J$. Thus $\bar{\alpha}(0)=\alpha(0) \leq \bar{\alpha}(T)$ and $\bar{\beta}(0)=\beta(0) \geq \bar{\beta}(T)$.

(i) If $\alpha(0) \leq \alpha(T)$, then

$$
\begin{aligned}
(\bar{\alpha}(\theta(t)))^{\prime} & +M \bar{\alpha}+\sum_{i=1}^{q} N_{i} \bar{\alpha}\left(\varphi_{i}(t)\right)=(\alpha(\theta(t)))^{\prime} \\
& +M \alpha(t)+\sum_{i=1}^{q} N_{i} \alpha\left(\varphi_{i}(t)\right) \leq \sigma(t), t \in J_{1}, \\
\Delta \bar{\alpha}\left(t_{k}\right)= & \Delta \alpha\left(t_{k}\right) \leq-L_{k} \alpha\left(t_{k}\right)+\gamma_{k}-a_{k} \leq-L_{k} \alpha\left(t_{k}\right)+\gamma_{k}-L_{k} a_{k} \\
= & -L_{k}\left[\alpha\left(t_{k}\right)+a_{k}\right]+\gamma_{k}=-L_{k} \bar{\alpha}\left(t_{k}\right)+\gamma_{k}, \quad k=1, \ldots, p,
\end{aligned}
$$

and add to $\bar{\alpha}(0) \leq \bar{\alpha}(T)$.

(ii) If $\alpha(0)>\alpha(T)$, then

$$
\begin{aligned}
& (\bar{\alpha}(\theta(t)))^{\prime}+M \bar{\alpha}(t)+\sum_{i=1}^{q} N_{i} \bar{\alpha}\left(\varphi_{i}(t)\right)=(\alpha(\theta(t)))^{\prime}+M \alpha(t) \\
& +\sum_{i=1}^{q} N_{i} \alpha\left(\varphi_{i}(t)\right)+\frac{\theta^{\prime}(t)+M t+\sum_{i=1}^{q} N_{i} \varphi_{i}(t)}{T}(\alpha(0)-\alpha(T)) \\
& \leq \sigma(t), \quad t \in J_{1}, \\
& \Delta \bar{\alpha}\left(t_{k}\right)=\Delta \alpha\left(t_{k}\right) \leq-L_{k} \bar{\alpha}\left(t_{k}\right)+\gamma_{k}, \quad k=1, \ldots, p,
\end{aligned}
$$

and add to $\bar{\alpha}(0) \leq \bar{\alpha}(T)$.

Thus, in either case, $\bar{\alpha}$ is a classical lower solution for (3.1). The same arguments show that $\bar{\beta}$ is a classical upper solution for (3.1). 
Now, we consider the function $m=\bar{\alpha}-\bar{\beta}$.

$$
\begin{aligned}
& (m(\theta(t)))^{\prime}+M m(t)+\sum_{i=1}^{q} N_{i} m\left(\varphi_{i}(t)\right) \\
& =\left[(\bar{\alpha}(\theta(t)))^{\prime}+M \bar{\alpha}(t)+\sum_{i=1}^{q} N_{i} \bar{\alpha}\left(\varphi_{i}(t)\right)\right] \\
& -\left[(\bar{\beta}(\theta(t)))^{\prime}+M \bar{\beta}(t)+\sum_{i=1}^{q} N_{i} \bar{\beta}\left(\varphi_{i}(t)\right)\right] \\
& \leq \sigma(t)-\sigma(t)=0, \quad t \in J_{1}, \\
& \Delta m\left(t_{k}\right)=\Delta \bar{\alpha}\left(t_{k}\right)-\Delta \bar{\beta}\left(t_{k}\right) \leq\left[-L_{k} \bar{\alpha}\left(t_{k}\right)+\gamma_{k}\right]-\left[-L_{k} \bar{\beta}\left(t_{k}\right)+\gamma_{k}\right] \\
& =-L_{k} m\left(t_{k}\right), \quad k=1, \ldots, p, \\
& m(0)=\bar{\alpha}(0)-\bar{\beta}(0) \leq \bar{\alpha}(T)-\bar{\beta}(T)=m(T) .
\end{aligned}
$$

Using Lemma 2 , we get $m \leq 0$ on $J$, i.e., $\bar{\alpha} \leq \bar{\beta}$ on $J$.

Thus, we have completed the proof of Theorem 1.

\section{Existence for nonlinear problem}

In this section, we establish the existence criteria for solutions of (1.1) by the lower and upper solutions and the monotone iterative technique.

Theorem 2. Suppose that there exist $\alpha, \beta \in \Omega$ such that

(D1) $\alpha$ and $\beta$ are lower and upper solutions for (1.1) with $\alpha \leq \beta$;

(D2) $f\left(t, x_{2}, y_{12}, \ldots, y_{q 2}\right)-f\left(t, x_{1}, y_{11}, \ldots, y_{q 1}\right) \geq-M\left(x_{2}-x_{1}\right)$

$$
\begin{aligned}
& -\sum_{i=1}^{q} N_{i}\left(y_{i 2}-y_{i 1}\right) \text { for every } t \in J_{1}, \alpha \leq x_{1} \leq x_{2} \leq \beta, \\
& \alpha\left(\varphi_{i}(t)\right) \leq y_{i 1}\left(\varphi_{i}(t)\right) \leq y_{i 2}\left(\varphi_{i}(t)\right) \leq \beta\left(\varphi_{i}(t)\right)(i=1, \ldots, q) ;
\end{aligned}
$$

(D3) $I_{k}(x)-I_{k}(y) \geq-L_{k}(x-y)$ for $\alpha\left(t_{k}\right) \leq y\left(t_{k}\right) \leq x\left(t_{k}\right) \leq \beta\left(t_{k}\right)$,

$$
k=1, \ldots, p \text {. }
$$

Also assume that (A4) holds. Then there exist monotone sequence $\left\{\bar{\alpha}_{n}(t)\right\}$, $\left\{\bar{\beta}_{n}(t)\right\}$ with $\bar{\alpha}_{0}=\bar{\alpha}, \bar{\beta}_{0}=\bar{\beta}$, where $\bar{\alpha}, \bar{\beta}$ are defined by (3.3) and (3.4), such that $\lim _{n \rightarrow \infty} \bar{\alpha}_{n}(t)=\rho(t)$ and $\lim _{n \rightarrow \infty} \bar{\beta}_{n}(t)=\psi(t)$ uniformly hold on $J$, where $\rho(t), \psi(t)$ are minimal and maximal solutions of (1.1), respectively. 
Proof. We shall prove the Theorem in the following three steps.

Step 1. It is evident that $\alpha \leq \bar{\alpha}$ and $\beta \leq \bar{\beta}$ on $J$. Thus $\alpha(0)=\bar{\alpha}(0) \leq \bar{\alpha}(T)$ and $\bar{\beta}(0)=\beta(0) \geq \bar{\beta}(T)$.

Let the function $m=\bar{\alpha}-\bar{\beta}$, then $m(0)=\bar{\alpha}(0)-\bar{\beta}(0) \leq \bar{\alpha}(T)-\bar{\beta}(T)=$ $m(T)$. Next, we consider two cases.

Case 1. $\alpha(0)>\alpha(T)$ and $\beta(0)<\beta(T)$.

Firstly, by (D2), we get

$$
\begin{aligned}
& (m(\theta(t)))^{\prime}+M m(t)+\sum_{i=1}^{q} N_{i} m\left(\varphi_{i}(t)\right) \\
& =\left[(\alpha(\theta(t)))^{\prime}+M \alpha(t)+\sum_{i=1}^{q} N_{i} \alpha\left(\varphi_{i}(t)\right)+\frac{\theta^{\prime}(t)+M t+\sum_{i=1}^{q} N_{i} \varphi_{i}(t)}{T}(\alpha(0)-\alpha(T))\right] \\
& \quad-\left[(\beta(\theta(t)))^{\prime}+M \beta(t)+\sum_{i=1}^{q} N_{i} \beta\left(\varphi_{i}(t)\right)-\frac{\theta^{\prime}(t)+M t+\sum_{i=1}^{q} N_{i} \varphi_{i}(t)}{T}(\beta(T)-\beta(0))\right] \\
& \leq\left[f\left(t, \alpha(t), \alpha\left(\varphi_{1}(t)\right), \ldots, \alpha\left(\varphi_{q}(t)\right)\right)-f\left(t, \beta(t), \beta\left(\varphi_{1}(t)\right), \ldots, \beta\left(\varphi_{q}(t)\right)\right)\right] \\
& \quad-\left[M(\beta(t)-\alpha(t))+\sum_{i=1}^{q} N_{i}\left(\beta\left(\varphi_{i}(t)\right)-\alpha\left(\varphi_{i}(t)\right)\right)\right] \\
& \leq 0, t \in J_{1} .
\end{aligned}
$$

Again, by (D3), we obtain

$$
\begin{aligned}
& \Delta m\left(t_{k}\right)=\Delta \bar{\alpha}\left(t_{k}\right)-\Delta \bar{\beta}\left(t_{k}\right)=\Delta \alpha\left(t_{k}\right)-\Delta \beta\left(t_{k}\right) \\
& \quad \leq\left[I_{k}\left(\alpha\left(t_{k}\right)\right)-L_{k} a_{k}\right]-\left[I_{k}\left(\beta\left(t_{k}\right)\right)+L_{k} b_{k}\right] \\
& \quad \leq-L_{k}\left[\alpha\left(t_{k}\right)-\beta\left(t_{k}\right)\right]-L_{k} a_{k}-L_{k} b_{k} \leq-L_{k} m\left(t_{k}\right), \quad k=1, \ldots, p .
\end{aligned}
$$

Finally, add to $m(0) \leq m(T)$. Using Lemma $2, m(t) \leq 0$ on $J$, i.e., $\bar{\alpha} \leq \bar{\beta}$ on $J$.

It follows that

$$
\begin{aligned}
(\bar{\alpha}(\theta(t)))^{\prime} & =(\alpha(\theta(t)))^{\prime}+\frac{\theta^{\prime}(t)}{T}[\alpha(0)-\alpha(T)] \\
& \leq f\left(t, \alpha(t), \alpha\left(\varphi_{1}(t)\right), \ldots, \alpha\left(\varphi_{q}(t)\right)\right)-\frac{M t+\sum_{i=1}^{q} N_{i} \varphi_{i}(t)}{T}[\alpha(0)-\alpha(T)] .
\end{aligned}
$$


Since $\alpha \leq \bar{\alpha} \leq \beta$, by (D2), we get

$$
\begin{aligned}
& f\left(t, \bar{\alpha}(t), \bar{\alpha}\left(\varphi_{1}(t)\right), \ldots, \bar{\alpha}\left(\varphi_{q}(t)\right)\right)-f\left(t, \alpha(t), \alpha\left(\varphi_{1}(t)\right), \ldots, \alpha\left(\varphi_{q}(t)\right)\right) \\
& \quad \geq-M[\bar{\alpha}(t)-\alpha(t)]-\sum_{i=1}^{q} N_{i}\left[\bar{\alpha}\left(\varphi_{i}(t)\right)-\alpha\left(\varphi_{i}(t)\right)\right] .
\end{aligned}
$$

Then

$$
\begin{aligned}
& (\bar{\alpha}(\theta(t)))^{\prime} \leq f\left(t, \bar{\alpha}(t), \bar{\alpha}\left(\varphi_{1}(t)\right), \ldots, \bar{\alpha}\left(\varphi_{q}(t)\right)\right)+M[\bar{\alpha}(t)-\alpha(t)] \\
& \quad+\sum_{i=1}^{q} N_{i}\left[\bar{\alpha}\left(\varphi_{i}(t)\right)-\alpha\left(\varphi_{i}(t)\right)\right]-\frac{M t+\sum_{i=1}^{q} N_{i} \varphi_{i}(t)}{T}[\alpha(0)-\alpha(T)] \\
& =f\left(t, \bar{\alpha}(t), \bar{\alpha}\left(\varphi_{1}(t)\right), \ldots, \bar{\alpha}\left(\varphi_{q}(t)\right)\right) \\
& \quad+\frac{M t+\sum_{i=1}^{q} N_{i} \varphi_{i}(t)}{T}[\alpha(0)-\alpha(T)]-\frac{M t+\sum_{i=1}^{q} N_{i} \varphi_{i}(t)}{T}[\alpha(0)-\alpha(T)] \\
& =f\left(t, \bar{\alpha}(t), \bar{\alpha}\left(\varphi_{1}(t)\right), \ldots, \bar{\alpha}\left(\varphi_{q}(t)\right)\right) .
\end{aligned}
$$

From (D3), we get

$$
\begin{aligned}
\Delta \bar{\alpha}\left(t_{k}\right)= & \Delta \alpha\left(t_{k}\right) \leq I_{k}\left(\alpha\left(t_{k}\right)\right)-\frac{L_{k} t_{k}}{T}[\alpha(0)-\alpha(T)] \\
& \leq I_{k}\left(\bar{\alpha}\left(t_{k}\right)\right)+L_{k}\left[\bar{\alpha}\left(t_{k}\right)-\alpha\left(t_{k}\right)\right]-\frac{L_{k} t_{k}}{T}[\alpha(0)-\alpha(T)] \\
= & I_{k}\left(\bar{\alpha}\left(t_{k}\right)\right) .
\end{aligned}
$$

Case 2. $\alpha(0) \leq \alpha(T)$ and $\beta(0) \geq \beta(T)$. In this case, it is trivial that we get (4.1) and (4.2).

Thus, in either case, $\bar{\alpha}$ is a classical lower solution. Similarly, $\bar{\beta}$ is a classical upper solution. Moreover $[\bar{\alpha}, \bar{\beta}] \subseteq[\alpha, \beta]$.

Step 2. For any $\eta \in[\bar{\alpha}, \bar{\beta}]$, we consider

$$
\left\{\begin{array}{l}
(u(\theta(t)))^{\prime}+M u(t)+\sum_{i=1}^{q} N_{i} u\left(\varphi_{i}(t)\right)=M \eta(t)+\sum_{i=1}^{q} N_{i} \eta\left(\varphi_{i}(t)\right) \\
\quad+f\left(t, \eta(t), \eta\left(\varphi_{1}(t)\right), \ldots, \eta\left(\varphi_{q}(t)\right)\right), \quad t \in J_{1}, \\
\Delta u\left(t_{k}\right)+L_{k} u\left(t_{k}\right)=I_{k}\left(\eta\left(t_{k}\right)\right)+L_{k} \eta\left(t_{k}\right), \quad k=1, \ldots, p, \\
u(0)=u(T) .
\end{array}\right.
$$


Then, by Theorem 1, (4.3) has a unique solution $u \in \Omega$.

Define operator $A$ by $u=A \eta$. Then $A$ possesses the following properties:

(E1) $\bar{\alpha} \leq A \bar{\alpha}, \bar{\beta} \geq A \bar{\beta}$;

(E2) $A \eta_{1} \leq A \eta_{2}$ for $\eta_{1}, \eta_{2} \in[\bar{\alpha}, \bar{\beta}]$ with $\eta_{1} \leq \eta_{2}$.

Firstly, let $m=\bar{\alpha}-\bar{\alpha}_{1}$, where $\bar{\alpha}_{1}=A \bar{\alpha}$. Then we get

$$
\begin{aligned}
& (m(\theta(t)))^{\prime}=(\bar{\alpha}(\theta(t)))^{\prime}-\left(\bar{\alpha}_{1}(\theta(t))\right)^{\prime} \\
& \quad \leq f\left(t, \bar{\alpha}(t), \bar{\alpha}\left(\varphi_{1}(t)\right), \ldots, \bar{\alpha}\left(\varphi_{q}(t)\right)\right)+M \bar{\alpha}_{1}(t)+\sum_{i=1}^{q} N_{i} \bar{\alpha}_{1}\left(\varphi_{i}(t)\right) \\
& \quad-M \bar{\alpha}(t)-\sum_{i=1}^{q} N_{i} \bar{\alpha}\left(\varphi_{i}(t)\right)-f\left(t, \bar{\alpha}(t), \bar{\alpha}\left(\varphi_{1}(t)\right), \ldots, \bar{\alpha}\left(\varphi_{q}(t)\right)\right) \\
& \quad=-M\left[\bar{\alpha}(t)-\bar{\alpha}_{1}(t)\right]-\sum_{i=1}^{q} N_{i}\left[\bar{\alpha}_{1}\left(\varphi_{i}(t)\right)-\bar{\alpha}\left(\varphi_{i}(t)\right)\right] \\
& \quad=-M m(t)-\sum_{i=1}^{q} N_{i} m\left(\varphi_{i}(t)\right), t \in J_{1}, \\
& \Delta m\left(t_{k}\right)=\Delta \bar{\alpha}\left(t_{k}\right)-\Delta \bar{\alpha}_{1}\left(t_{k}\right) \\
& \quad \leq I_{k}\left(\bar{\alpha}\left(t_{k}\right)\right)-I_{k}\left(\bar{\alpha}\left(t_{k}\right)\right)-L_{k} \bar{\alpha}\left(t_{k}\right)+L_{k} \bar{\alpha}_{1}\left(t_{k}\right) \\
& \quad=-L_{k} m\left(t_{k}\right), k=1, \ldots, p, \\
& m(0)=\bar{\alpha}(0)-\bar{\alpha}_{1}(0) \leq \bar{\alpha}(T)-\bar{\alpha}_{1}(T)=m(T) .
\end{aligned}
$$

By Lemma 2, we have $m(t) \leq 0$ on $J$, i.e., $\bar{\alpha} \leq A \bar{\alpha}$. Similarly, we get $\bar{\beta} \geq A \bar{\beta}$.

Next, set $v_{1}=A \eta_{1}$ and $v_{2}=A \eta_{2}$, where $\eta_{1}, \eta_{2} \in[\bar{\alpha}, \bar{\beta}]$ with $\eta_{1} \leq \eta_{2}$. Let $m=v_{1}-v_{2}$. By (D2), (D3) and (4.3), we get

$$
\begin{aligned}
&(m(\theta(t)))^{\prime}=\left(v_{1}(\theta(t))\right)^{\prime}-\left(v_{2}(\theta(t))\right)^{\prime} \\
&= {\left[-M v_{1}(t)-\sum_{i=1}^{q} N_{i} v_{1}\left(\varphi_{i}(t)\right)+f\left(t, \eta_{1}(t), \eta_{1}\left(\varphi_{1}(t)\right), \ldots, \eta_{1}\left(\varphi_{q}(t)\right)\right)\right.} \\
&\left.+M \eta_{1}(t)+\sum_{i=1}^{q} N_{i} \eta_{1}\left(\varphi_{i}(t)\right)\right]-\left[-M v_{2}(t)-\sum_{i=1}^{q} N_{i} v_{2}\left(\varphi_{i}(t)\right)\right. \\
&\left.+f\left(t, \eta_{2}(t), \eta_{2}\left(\varphi_{1}(t)\right), \ldots, \eta_{2}\left(\varphi_{q}(t)\right)\right)+M \eta_{2}(t)+\sum_{i=1}^{q} N_{i} \eta_{2}\left(\varphi_{i}(t)\right)\right] \\
&=-M m(t)-\sum_{i=1}^{q} N_{i} m\left(\varphi_{i}(t)\right)+\left[f\left(t, \eta_{1}(t), \eta_{1}\left(\varphi_{1}(t)\right), \ldots, \eta_{1}\left(\varphi_{q}(t)\right)\right)\right. \\
&\left.-f\left(t, \eta_{2}(t), \eta_{2}\left(\varphi_{1}(t)\right), \ldots, \eta_{2}\left(\varphi_{q}(t)\right)\right)\right] \\
&-\left[M\left(\eta_{2}(t)-\eta_{1}(t)\right)+\sum_{i=1}^{q} N_{i}\left(\eta_{2}\left(\varphi_{i}(t)\right)-\eta_{1}\left(\varphi_{i}(t)\right)\right)\right]
\end{aligned}
$$




$$
\begin{aligned}
& \leq-M m(t)-\sum_{i=1}^{q} N_{i} m\left(\varphi_{i}(t)\right), \quad t \in J_{1}, \\
& \Delta m\left(t_{k}\right)=\Delta v_{1}\left(t_{k}\right)-\Delta v_{2}\left(t_{k}\right) \\
& =\left[-L_{k} v_{1}\left(t_{k}\right)+I_{k}\left(\eta_{1}\left(t_{k}\right)\right)+L_{k} \eta_{1}\left(t_{k}\right)\right] \\
& \quad-\left[-L_{k} v_{2}\left(t_{k}\right)+I_{k}\left(\eta_{2}\left(t_{k}\right)\right)+L_{k} \eta_{2}\left(t_{k}\right)\right] \\
& \leq-L_{k} m\left(t_{k}\right), \quad k=1, \ldots, p, \\
& m(0)=m(T) .
\end{aligned}
$$

By Lemma 2 , we get $m(t) \leq 0$ on $J$, i.e., $v_{1} \leq v_{2}$ on $J$. Then $A \eta_{1} \leq A \eta_{2}$ for $\eta_{1}, \eta_{2} \in[\bar{\alpha}, \bar{\beta}]$ with $\eta_{1} \leq \eta_{2}$.

Step 3. Define the sequence $\left\{\bar{\alpha}_{n}(t)\right\},\left\{\bar{\beta}_{n}(t)\right\}$ by $\bar{\alpha}_{n+1}=A \bar{\alpha}_{n}, \bar{\beta}_{n+1}=A \bar{\beta}_{n}$, $\bar{\alpha}_{0}=\bar{\alpha}, \bar{\beta}_{0}=\bar{\beta}$. From (E1) and (E2), we get

$$
\bar{\alpha}_{0} \leq \bar{\alpha}_{1} \leq \ldots \leq \bar{\alpha}_{n} \leq \bar{\beta}_{n} \leq \ldots \leq \bar{\beta}_{1}=\bar{\beta}_{0}, \quad \forall n \in N .
$$

Thus it is immediate to verify that

$$
\lim _{n \rightarrow \infty} \bar{\alpha}_{n}(t)=\rho(t) \quad \text { and } \quad \lim _{n \rightarrow \infty} \bar{\beta}_{n}(t)=\psi(t)
$$

uniformly hold on $J$.

We consider the equation

$$
\left\{\begin{array}{l}
\left(\bar{\alpha}_{n+1}(\theta(t))\right)^{\prime}+M \bar{\alpha}_{n+1}(t)+\sum_{i=1}^{q} N_{i} \bar{\alpha}_{n+1}\left(\varphi_{i}(t)\right)=M \bar{\alpha}_{n}(t) \\
\quad+\sum_{i=1}^{q} N_{i} \bar{\alpha}_{n}\left(\varphi_{i}(t)\right)+f\left(t, \bar{\alpha}_{n}(t), \bar{\alpha}_{n}\left(\varphi_{1}(t)\right), \ldots, \bar{\alpha}_{n}\left(\varphi_{q}(t)\right)\right), t \in J_{1}, \\
\Delta \bar{\alpha}_{n+1}\left(t_{k}\right)+L_{k} \bar{\alpha}_{n+1}\left(t_{k}\right)=I_{k}\left(\bar{\alpha}_{n}\left(t_{k}\right)\right)+L_{k} \bar{\alpha}_{n}\left(t_{k}\right), \quad k=1, \ldots, p, \\
\bar{\alpha}_{n+1}(0)=\bar{\alpha}_{n+1}(T),
\end{array}\right.
$$

and pass to the limit when $n$ tends to $\infty$. Thus we obtain that $\rho$ is a solution of (1.1). Analogously, $\psi$ is also a solution of (1.1).

Finally, let $u$ be any solution of (1.1) on $[\bar{\alpha}, \bar{\beta}]$. Clearly $\bar{\alpha}_{0} \leq u$. Assume $\bar{\alpha}_{n} \leq u$. We get that $\bar{\alpha}_{n+1} \leq u$ by considering the function $m=u-\bar{\alpha}_{n+1}$ and using Lemma 3 again. Then by passing to the limit, we conclude $\rho \leq u$ on $J$. Similarly, $u \leq \psi$ on $J$. Then $\rho(t), \psi(t)$ are minimal and maximal solutions of (1.1), respectively. Thus, we have completed the proof of Theorem 2. 


\section{An example}

Now we consider the equation

$$
\left\{\begin{array}{l}
\left(u\left(t^{2}\right)\right)^{\prime}=-\frac{1}{24} u^{2}(t)-\frac{1}{36}\left[u\left(\frac{1}{3} t\right)+u(\sqrt{t})+u\left(\frac{2}{3} t\right)\right] \\
\quad+\frac{1}{24} t, \quad t \in[0,1], t \neq \zeta_{1}=\frac{1}{2}, \\
\Delta u\left(t_{1}\right)=-\frac{1}{2} u\left(t_{1}\right), \quad t_{1}=\frac{1}{4}, \\
u(0)=u(1) .
\end{array}\right.
$$

Firstly, it is obvious that $\alpha=0$ is a classical lower solution for (5.1). Certainly $\alpha=0$ is a lower solution. Similarly $\beta=1$ is an upper solution. Moreover $\alpha \leq \beta$ on $J=[0,1]$. Next,

$$
\begin{aligned}
f(t & \left., x_{2}, y_{12}, y_{22}, y_{32}\right)-f\left(t, x_{1}, y_{11}, y_{21}, y_{31}\right) \\
& =-\frac{1}{24}\left(x_{2}^{2}-x_{1}^{2}\right)-\frac{1}{36} \sum_{i=1}^{3}\left(y_{i 2}-y_{i 1}\right) \\
& =-\frac{1}{24}\left(x_{2}+x_{1}\right)\left(x_{2}-x_{1}\right)-\frac{1}{36} \sum_{i=1}^{3}\left(y_{i 2}-y_{i 1}\right) \\
& \geq-\frac{1}{12}\left(x_{2}-x_{1}\right)-\frac{1}{36} \sum_{i=1}^{3}\left(y_{i 2}-y_{i 1}\right),
\end{aligned}
$$

for $\alpha \leq x_{1} \leq x_{2} \leq \beta, \alpha\left(\frac{1}{3} t\right) \leq y_{11}\left(\frac{1}{3} t\right) \leq y_{12}\left(\frac{1}{3} t\right) \leq \beta\left(\frac{1}{3} t\right), \alpha(\sqrt{t}) \leq$ $y_{21}(\sqrt{t}) \leq y_{22}(\sqrt{t}) \leq \beta(\sqrt{t}), \alpha\left(\frac{2}{3} t\right) \leq y_{31}\left(\frac{2}{3} t\right) \leq y_{32}\left(\frac{2}{3} t\right) \leq \beta\left(\frac{2}{3} t\right)$, where $M=\frac{1}{12}, N_{1}=N_{2}=N_{3}=\frac{1}{36}$. Further, $I_{1}(x)-I_{1}(y)=-\frac{1}{2} x+\frac{1}{2} y \geq$ $-\frac{1}{2}(x-y)$, for $\alpha\left(\frac{1}{4}\right) \leq y\left(\frac{1}{4}\right) \leq x\left(\frac{1}{4}\right) \leq \beta\left(\frac{1}{4}\right)$, where $0 \leq L_{1}=\frac{1}{2}<1$. Finally,

$$
\begin{gathered}
\left(M+\sum_{i=1}^{3} N_{i}\right) \int_{0}^{T} \prod_{s<\zeta_{k}<T}\left(1-L_{k}\right) d s=\frac{1}{6} \int_{0}^{1} \prod_{s<\zeta_{k}<T}\left(1-L_{k}\right) d s \\
=\frac{1}{6}\left[\int_{0}^{1 / 2} \frac{1}{2} d s+\int_{1 / 2}^{1} d s\right]=\frac{1}{8} \leq \frac{1}{4}=\prod_{k=1}^{p}\left(1-L_{k}\right)^{2} .
\end{gathered}
$$

Then all the conditions of Theorem 2 are satisfied. Thus (5.1) has minimal and maximal solutions in $[\alpha, \beta]$.

In addition, we consider $\beta_{1}(t)=\frac{t}{100}+\frac{99}{100}, t \in[0,1]$. Clearly $\beta_{1}(0)<\beta_{1}(1)$, then $b(t)=\frac{t}{1200}+\frac{1}{3600}\left(\frac{1}{3} t+\sqrt{t}+\frac{2}{3} t\right)+\frac{t}{50}$ and $b_{1}=\frac{1}{400}$. We still take 


$$
\begin{aligned}
& M= \frac{1}{12}, N_{1}=N_{2}=N_{3}=\frac{1}{36} . \text { From } \\
& 0 \geq-\frac{1}{24}\left[\left(\frac{t-1}{100}\right)^{2}+\frac{9800}{100^{2}}+\left(\frac{99}{50}-t\right)\right] \\
&=-\frac{1}{24}\left[\left(\frac{t}{100}\right)^{2}-\frac{t}{50} \times \frac{1}{100}+\frac{1}{100^{2}}+\frac{9800}{100^{2}}-t+2 \times \frac{99}{100}\right] \\
&=-\frac{1}{24}\left[\left(\frac{t}{100}\right)^{2}+\frac{t}{50} \times \frac{99}{100}+\left(\frac{99}{100}\right)^{2}-t-\frac{t}{50}+2 \times \frac{99}{100}\right] \\
&=-\frac{1}{24}\left(\frac{1}{100} t+\frac{99}{100}\right)^{2}-\frac{1}{36} \times 3 \times \frac{99}{100}+\frac{1}{24} t+\frac{t}{1200}, \\
& \frac{t}{50} \geq-\frac{1}{24} \beta_{1}^{2}(t)-\frac{1}{36}\left[\left(\frac{1}{100} \times \frac{1}{3} t+\frac{99}{100}\right)+\left(\frac{1}{100} \times \sqrt{t}+\frac{99}{100}\right)\right. \\
&\left.+\left(\frac{1}{100} \times \frac{2}{3} t+\frac{99}{100}\right)\right]+\frac{1}{24} t+\left[\frac{t}{1200}+\frac{1}{3600}\left(\frac{1}{3} t+\sqrt{t}+\frac{2}{3} t\right)+\frac{t}{50}\right],
\end{aligned}
$$

we have $\left(\beta_{1}\left(t^{2}\right)\right)^{\prime} \geq-\frac{1}{24} \beta_{1}^{2}(t)-\frac{1}{36}\left[\beta_{1}\left(\frac{1}{3} t\right)+\beta_{1}(\sqrt{t})+\beta_{1}\left(\frac{2}{3} t\right)\right]+\frac{1}{24} t+b(t)$. From $0 \geq-\frac{1}{2}\left(\frac{1}{100} \times \frac{1}{4}+\frac{99}{100}\right)+\frac{1}{2} \times \frac{1}{400}$, we get $\Delta \beta_{1}\left(\frac{1}{4}\right) \geq I_{1}\left(\beta_{1}\left(\frac{1}{4}\right)\right)+L_{1} b_{1}$.

These show that $\beta_{1}(t)$ is an upper solution for (5.1). Moreover $\alpha \leq \beta_{1}$ on $J$. Similarly, we get the existence of monotone sequence that approximate the extremal solutions for $(5.1)$ in $\left[\alpha, \beta_{1}\right]$.

Acknowledgements. The authors are very grateful to the referee for useful remarks and interesting comments.

\section{REFERENCES}

[1] D.D. Bainov and P.S. Simeonov, Impulsive Differential Equations: Periodic Solutions and Applications. Longman Scientific and Technical, Harlow (1993).

[2] V. Lakshmikantham, D. Bainov and P. Simeonov, Theory of Impulsive Differential Equations. World Scientific, Singapore (1989).

[3] A. Halanay and D. Wexler, Qualitative Theory of Impulse Systems. Editura Academic Republic Socialiste Romania, Bucuresti (1968).

[4] A.M. Samoilenko and N.A. Perestyuk, Impulsive Differential Equations [M]. World Scientific, Singapore (1995).

[5] X.L. Fu, B.Q. Yan and Y.S. Liu, Theory of impulsive differential system. Science Press, Beijing (2005).

[6] R. Bellman, Mathematical Methods in Medicine. World Scientific, Singapore (1983). 
[7] M. Yao, A. Zhao and J. Yan, Periodic boundary value problems for second-order impulsive differential equations. Nonlinear Anal., 70 (2009), 262-273.

[8] Y. Liu, Further results on periodic boundary value problems for nonlinear first order impulsive functional differential equations. J. Math. Anal. Appl., 327 (2007), 435-452.

[9] J. Li and J. Shen, Periodic boundary value problems for delay differential equations with impulses. J. Comput. Appl. Math., 193 (2006), 563-573.

[10] J. Li and J. Shen, Periodic boundary value problems for impulsive integro-differential equations of mixed type. J. Appl. Math. Comput., 183 (2006), 890-902.

[11] J. Li and J. Shen, Periodic boundary value problems for impulsive differential-difference equations. Indian J. Pure Appl. Math., 35 (2004), 1265-1277.

[12] Z. He and X. Ze, Monotone iterative technique for impulsive integro-differential equations with periodic boundary conditions. Comput. Math. Appl., 48 (2004), 73-84.

[13] J. Shen, New maximum principles for first-order impulsive periodic boundary value problems. Appl. Math. Lett., 16 (2003), 105-112.

[14] G. Song and X. Zhu, Extremal solutions of periodic boundary value problems for first order integro-differential equations of mixed type. J. Math. Anal. Appl., 300 (2004), 1-11.

[15] Y.-K. Chang, A. Anguraj and M. Mallika Arjunan, Existence results for impulsive neutral functional differential equations with infinite delay. Nonlinear Analysis: Hybrid Systems, 2 (2008), 209-218.

[16] A. Anguraj and K. Karthikeyan, Existence of solutions for impulsive neutral functional differential equations with nonlocal conditions. Nonlinear Anal., 70 (2009), 2717-2721.

[17] C. Cuevas, E. Hernández and M. Rabelo, The existence of solutions for impulsive neutral functional differential equations. Comput. Math. Appl., 58 (2009), 744-757.

[18] J.Y. Park, K. Balachandran and N. Annapoorani, Existence results for impulsive neutral functional integrodifferential equations with infinite delay. Nonlinear Anal., 71 (2009), 3152-3162.

[19] Eduardo Hernández Morales, Hernán R. Henríquez and Mark A. McKibben, Existence results for abstract impulsive second-order neutral functional differential equations. Nonlinear Anal., 70 (2009), 2736-2751. 